\title{
Reduction in adjacent-segment degeneration after multilevel posterior lumbar interbody fusion with proximal DIAM implantation
}

\author{
Kang Lu, MD, PhD, ${ }^{1}$ Po-Chou Liliang, MD, ${ }^{1}$ Hao-Kuang Wang, MD, ${ }^{1}$ Cheng-Loong Liang, MD, PhD, ${ }^{1}$ \\ Jui-Sheng Chen, MD, ${ }^{1}$ Tai-Been Chen, PhD, ${ }^{2}$ Kuo-Wei Wang, MD, ${ }^{1}$ and Han-Jung Chen, MD, PhD ${ }^{1}$ \\ 'Department of Neurosurgery, E-Da Hospital, I-Shou University; and 2Department of Medical Imaging and Radiological Sciences, \\ I-Shou University, Kaohsiung, Taiwan
}

\begin{abstract}
OBJECT Multilevel long-segment lumbar fusion poses a high risk for future development of adjacent-segment degeneration (ASD). Creating a dynamic transition zone with an interspinous process device (IPD) proximal to the fusion has recently been applied as a method to reduce the occurrence of ASD. The authors report their experience with the Device for Intervertebral Assisted Motion (DIAM) implanted proximal to multilevel posterior lumbar interbody fusion (PLIF) in reducing the development of proximal ASD.

METHODS This retrospective study reviewed 91 cases involving patients who underwent 2-level (L4-S1), 3-level (L3-S1), or 4-level (L2-S1) PLIF. In Group A (42 cases), the patients received PLIF only, while in Group B (49 cases), an interspinous process device, a DIAM implant, was put at the adjacent level proximal to the PLIF construct. Bone resection at the uppermost segment of the PLIF was equally limited in the 2 groups, with preservation of the upper portion of the spinous process/lamina and the attached supraspinous ligament. Outcome measures included a visual analog scale (VAS) for low-back pain and leg pain and the Oswestry Disability Index (ODI) for functional impairment. Anteroposterior and lateral flexion/extension radiographs were used to evaluate the fusion status, presence and patterns of ASD, and mobility of the DIAM-implanted segment.
\end{abstract}

RESULTS Solid interbody fusion without implant failure was observed in all cases. Radiographic ASD occurred in 20 $(48 \%)$ of Group A cases and $3(6 \%)$ of Group B cases $(p<0.001)$. Among the patients in whom ASD was identified, 9 in Group A and 3 in Group B were symptomatic; of these patients, 3 in Group A and 1 in Group B underwent a second surgery for severe symptomatic ASD. At 24 months after surgery, Group A patients fared worse than Group B, showing higher mean VAS and ODI scores due to symptoms related to ASD. At the final follow-up evaluations, as reoperations had been performed to treat symptomatic ASD in some patients, significant differences no longer existed between the 2 groups. In Group B, flexion/extension mobility at the DIAM-implanted segment was maintained in 35 patients and restricted or lost in 14 patients, 5 of whom had already lost segmental flexion/extension mobility before surgery. No patient in Group B developed ASD at the segment proximal to the DIAM implant.

CONCLUSIONS Providing a dynamic transition zone with a DIAM implant placed immediately proximal to a multilevel PLIF construct was associated with a significant reduction in the occurrence of radiographic ASD, compared with PLIF alone. Given the relatively old age and more advanced degeneration in patients undergoing multilevel PLIF, this strategy appears to be effective in lowering the risk of clinical ASD and a second surgery subsequent to PLIF.

http://thejns.org/doi/abs/10.3171/2014.12.SPINE14666

KEY WORDS adjacent-segment degeneration; posterior lumbar interbody fusion; DIAM; interspinous process device; degenerative disease

\footnotetext{
ABBREVIATIONS ASD = adjacent-segment degeneration; DIAM = Device for Intervertebral Assisted Motion; IPD = interspinous process device; ODI = Oswestry Disability Index; PLIF = posterior lumbar interbody fusion; VAS = visual analog scale.

SUBMITTED July 12, 2014. ACCEPTED December 3, 2014.

INCLUDE WHEN CITING Published online May 1, 2015; DOI: 10.3171/2014.12.SPINE14666.

DISCLOSURE The authors report no conflict of interest concerning the materials or methods used in this study or the findings specified in this paper.
} 
A DJACENT-SEGMENT degeneration (ASD) is a welldocumented sequela of lumbar and lumbosacral fusions. The incidence of radiographically verified ASD reported in the literature varies from 5.6\% to $100 \% .^{13,16,17}$ A number of clinical observations indicate that the level proximal to fusion is more likely to undergo degenerative changes than the level distal to the fusion. ${ }^{16}$ ASD may manifest as disc degeneration, disc herniation, facet joint hypertrophy, stenosis, segmental instability, and spondylolisthesis. ${ }^{4,11,15,21}$ Symptomatic ASD, or adjacent-segment disease, remains a significant cause of postoperative morbidity and reoperation following lumbar arthrodesis.

Different preemptive measures have been used to reduce the development of ASD, including avoiding violation of the superior facet joint by pedicle screws, ${ }^{2}$ minimizing bone and ligament resection in laminectomy, ${ }^{8,12}$ and using semirigid, nonfusion instrumentation instead of rigid fixation..$^{10}$ In addition, the idea of applying a soft, nonrigid, dynamic stabilizing device adjacent to fusion as a means to prevent ASD has also been proposed ${ }^{7,8}$ Recently, applying an interspinous process device (IPD) at the unfused segment cephalad to pedicle screw instrumentation to create a dynamic transition zone has been reported., ${ }^{914}$ Although the preliminary results are appealing, the fusion segments in the reported cases were relatively short. The effectiveness of implanting an IPD proximal to long-segment multilevel fusion is not known. Nor is it clear whether the structural status at the implanted segment would limit or even adversely affect the use of an IPD.

Since 2009, we have been using a strategy of putting a silicon-based IPD, the DIAM (Device for Intervertebral Assisted Motion, Medtronic) implant, proximal to multilevel posterior lumbar interbody fusion (PLIF) constructs to prevent the development of ASD. In this study, we retrospectively reviewed and compared patients who received DIAM implantation and those who did not.

\section{Methods \\ Patient Population}

This retrospective study was based on a review of medical records approved by the ethics committee of E-Da Hospital. From March 2009 to February 2011, 91 patients with degenerative lumbar disease were treated with multilevel PLIF by the same surgeon (K.L.). The inclusion criteria were lumbar spinal stenosis, disc degeneration, and spondylolisthesis, alone or in combination, involving 2 to 4 disc segments including the L5-S1 level. Patients with previous spinal infection, trauma, or lumbar surgery, were excluded. All patients underwent 2-level (L4-S1) to 4-level (L2-S1) PLIF.

Prior to surgery, all patients had been treated with medication, activity modification, and physical therapy for at least 3 months. Indications for surgery included refractory low-back and leg pain, disabling intermittent claudication, and progression of neurological deficits attributable to lumbar stenosis.

Neutral anteroposterior, lateral flexion/extension radiographs and nonenhanced MRI of the lumbar spine were obtained in all patients.

\section{Surgical Procedures}

The patients were divided into 2 groups, according to whether a DIAM implant was placed immediately proximal to the fusion construct. Patients in Group A $(n=42)$ were treated with PLIF only, while those in Group B (n = 49) were treated with PLIF and DIAM implantation. To determine the feasibility of DIAM implantation, the uppermost disc segment of the planned fusion construct was evaluated based on the Pfirrmann grading system. ${ }^{19}$ If disc height loss was less than $50 \%$ and segmental flexion/ extension mobility was present, DIAM implantation was regarded as feasible. However, DIAM implantation was performed in some cases showing no obvious segmental flexion/extension mobility, as the status of the discs was regarded as suitable. The decision for DIAM implantation was made at the patient's discretion after getting a written form detailing the usage of the implant and thorough explanation from the operating surgeon. The number of fusion levels, and therefore, the level where the DIAM was implanted, were based on imaging findings.

PLIF procedures included laminectomy, medial facetectomy, excision of ligamenta flava, and discectomy, followed by interbody PEEK (polyetheretherketone) cage fusion and rigid pedicle screw fixation. In both groups, at the uppermost segment for PLIF, only the lower portions of the spinous process and lamina were resected, leaving at least half of the bony structure, facet joints, and the proximal supraspinous ligament intact (Fig. 1A and B). In Group B, the DIAM implant was placed between the remaining spinous process/lamina and the rostral adjacent counterparts under the supraspinous ligament, after removal of the interspinous ligament and the ligamentum flavum between them. If segmental stenosis existed at the DIAM-implanted level and decompression was deemed necessary, limited undercutting of the facet joints and removal of the ligamentum in the lateral recesses were performed as well, while the facet joints were preserved. The implant size was determined by inserting trials $(8$,
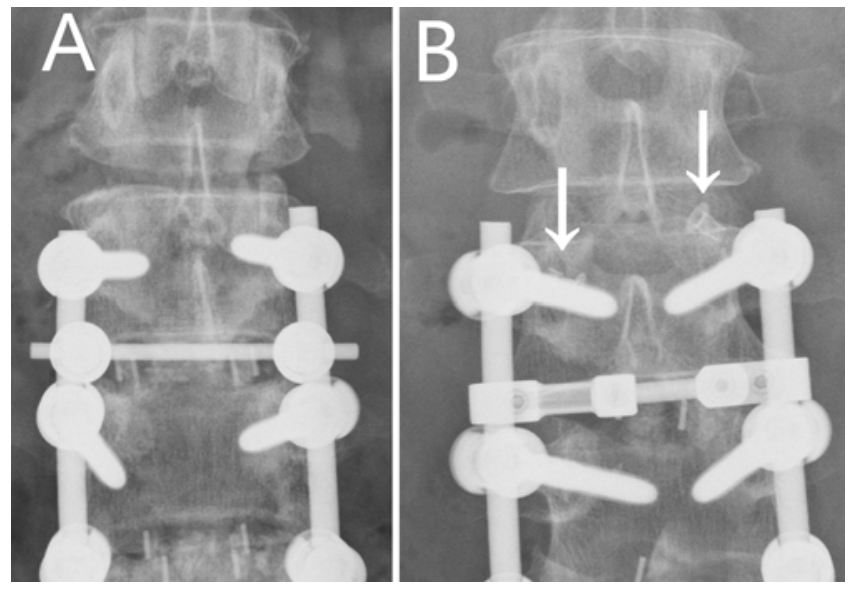

FIG. 1. KUB (kidney, ureter, bladder) radiographs of representative patients from Groups $A(A)$ and $B(B)$. In both groups, the spinous processes and laminae at the uppermost PLIF segment were only partially resected, leaving the upper portion and the attached supraspinatous ligament intact ( $A$ and $B$ ). In Group B, a DIAM implant, visualized by its 2 crimps (arrows) for tether fixation, was placed between the remaining spinous process/lamina at the proximal adjacent segment (B). 
10,12 , and $14 \mathrm{~mm}$ ) between the spinous processes under distraction. The implant was firmly anchored in place by 2 tethers passed around the 2 adjacent spinous processes (Fig. 1B).

\section{Clinical Evaluation}

All patients were evaluated before and at designated follow-up time points after surgery. Back and leg pain intensity and functional impairment were assessed with the visual analog scale (VAS) and the Oswestry Disability Index (ODI), respectively.

\section{Radiographic Evaluation}

Anteroposterior and lateral flexion/extension radiographs were routinely obtained during follow-up visits at 3 weeks and 3,6, and 12 months, and then annually thereafter. Radiographs taken at 3 weeks postoperatively and at the last follow-up were compared. Solid interbody fusion was defined as trabecular bone growth across the disc space within and around the cages, and absence of motion on flexion/extension radiographs. ${ }^{20}$ The radiographic criteria for ASD included the development of anterolisthesis, retrolisthesis due to hypermobility on flexion/extension, or loss of disc height, with or without intradiscal vacuum clefts and sclerosis along endplates (disc degeneration). ${ }^{6}$ The 2 disc spaces cephalad to the fusion construct were evaluated for signs of ASD. For Group B, the DIAM implant position and the presence or absence of flexion/extension segmental mobility were also observed.

Patients who sustained vertebral compression fractures involving the immediate proximal adjacent segment during follow-up were excluded to avoid confounding factors from the fractures. Patients showing radiographic evidence of ASD but reporting no symptoms were defined as having asymptomatic ASD. Those having radiographic ASD and associated symptoms attributable to the ASD were categorized as having symptomatic ASD.

An MRI study was obtained when reoperation for symptomatic ASD was anticipated. MRI criteria of ASD were spinal canal stenosis, facet hypertrophy, and/or disc degeneration at the adjacent segment(s) that had not been present initially. All imaging diagnoses were made by an independent radiologist.

\section{Statistical Analysis}

Clinical characteristics of the patients were compared between Groups A and B. The variables were expressed either as means with standard deviations (for continuous variables) or numbers with percentages (for categorical variables), and the results were compared by using an independent-sample t-test or Pearson chi-square or Fisher exact test when appropriate. To investigate the risk factors for poor outcome, those variables whose $p$ values were below 0.05 in bivariate analyses were included in the multivariable logistic regression analysis. All analyses were performed by using SPSS 15 (SPSS Inc.).

\section{Results}

\section{Patient Characteristics}

The patients' demographic characteristics, number of levels fused, and duration of follow-up are summarized in Table 1. There was no significant difference between the groups with respect to sex distribution and duration of follow-up. Group B patients (mean age $64.5 \pm 7.2$ years) were significantly older than Group A patients $(59.1 \pm 8.6$ years) $(\mathrm{p}=0.001)$. The proportion of patients who underwent 2-level (L4-S1) PLIF was significantly greater in Group A than in Group B ( $p=0.003)$, while there was no difference between groups in the proportion of patients who underwent 3-level (L3-S1) or 4-level (L2-S1) PLIF (Table 1).

\section{Radiographic Outcome}

The radiographic findings are summarized in Table 2. Radiographic ASD developed in 20 patients $(48 \%)$ in Group A (Fig. 2A and B) and in $3(6 \%)$ in Group B (p $<0.001$ ). Among these patients, 9 in Group A and 3 in Group B had ASD-associated symptoms $(\mathrm{p}=0.217)$. Reoperation for symptomatic ASD was performed in 3 patients in Group A and 1 in Group B ( $\mathrm{p}=0.332)$ (Table 2).

The angle between the upper L-1 and S-1 endplates was measured and defined as the lumbar lordotic angle. The mean pre- and postoperative lumbar lordotic angles were $41.5^{\circ} \pm 14.3^{\circ}$ and $36.2^{\circ} \pm 11.2^{\circ}$ in Group A and $40.6^{\circ} \pm$ $10.8^{\circ}$ and $34.7^{\circ} \pm 10.8^{\circ}$ in Group B, respectively. Significant differences existed between the pre- and postoperative lordotic angles in both groups ( $\mathrm{p} \leq 0.001)$. There were no differences between groups in the preoperative $(\mathrm{p}=$ $0.719)$ and postoperative lordotic angles $(\mathrm{p}=0.523)$.

The patterns of radiographic ASD in Group A included retrolisthesis (increased backward mobility on extension) in 9 cases, retrolisthesis with disc degeneration in 6, anterolisthesis in 3, anterolisthesis with disc degeneration in 1 , and disc degeneration in 1. The patterns in Group B were retrolisthesis with disc degeneration in 1 case and disc degeneration in 2 cases (Table 3). There was no radiographic ASD proximal to the DIAM segments in any Group B patient.

The Pfirrmann grades of the disc proximal to PLIF prior to surgery in all patients are summarized in Table 4. In $34(81 \%)$ of the Group A patients, and $22(45 \%)$ of the Group B patients, the discs were graded as Pfirrmann

\section{TABLE 1. Demographic characteristics of 91 patients treated with multilevel PLIF with (Group B) or without (Group A) DIAM implantation}

\begin{tabular}{lccc}
\hline \multicolumn{1}{c}{ Characteristic } & Group A $(n=42)$ & Group B $(n=49)$ & $p$ Value \\
\hline Age & & \\
\hline Mean \pm SD & $59.1 \pm 8.6$ & $64.5 \pm 7.2$ & 0.001 \\
\hline Range & $35-81$ & $46-78$ & \\
\hline Male/female & $14 / 28$ & $16 / 33$ & 0.945 \\
\hline No. of levels fused & & & \\
\hline 2 (L4-S1) & $21(50 \%)$ & $10(20 \%)$ & 0.003 \\
\hline $3($ L3-S1) & $17(40 \%)$ & $30(61 \%)$ & 0.048 \\
\hline $4($ L2-S1) & $4(10 \%)$ & $9(19 \%)$ & 0.229 \\
\hline Follow-up (mos) & & & \\
\hline Mean \pm SD & $41.5 \pm 8.6$ & $41.2 \pm 7.2$ & 0.687 \\
\hline Range & $24-48$ & $24-48$ & \\
\hline
\end{tabular}


TABLE 2. Radiographic outcome

\begin{tabular}{lccc}
\hline Characteristic & Group A $(n=42)$ & Group B $(n=49)$ & $p$ Value \\
\hline Radiographic ASD & & & $<0.001$ \\
\hline Yes & $20(48 \%)$ & $3(6 \%)$ & \\
\hline No & $22(52 \%)$ & $46(94 \%)$ & \\
\hline Symptomatic & & & 0.217 \\
\hline Yes & 9 & 3 & \\
\hline No & 11 & 0 & 0.332 \\
\hline Reoperation* & 3 & 1 & \\
\hline
\end{tabular}

* Performed for disabling symptoms and neurological dysfunction associated with severe ASD.

Grade I or II, while in 8 (19\%) of the Group A patients and $27(55 \%)$ of the Group B patients, the grades were III or IV (Table 4).

Table 5 further summarizes the preoperative Pfirrmann grades and final flexion/extension mobility of DIAM-implanted segments in Group B patients. Comparison with preoperative images showed that flexion/extension mobility at the DIAM-implanted segments was maintained in 34 patients (Fig. 2C and D) but was lost or became restricted in 15 patients, including the 3 Group B patients in whom ASD was identified (Table 5). Of the 15 patients showing no or restricted mobility at the final follow-up examinations, 5 actually had lost the mobility before surgery.

\section{Reoperations for ASD}

Among the patients with symptomatic ASD, 3 in Group $A$ and 1 in Group B underwent reoperation because of intractable pain and progressive claudication. The durations between the first and second operations were 25, 35, 48, and 31 months respectively. MRI showed severe stenosis at the adjacent levels in all of these patients. The second operations involved removal of previously placed pedicle screws and rods, decompression, fusion with pedicle fixation at the adjacent segments, and implantation of a DIAM implant proximal to the new fusion constructs. All of these patients reported symptom relief and functional improvement after the second surgery. Follow-up images did not show further ASD at either the DIAM-implanted segment or the level above.

The other patients with symptomatic ASD opted for nonsurgical treatment, although in 3 Group A patients, symptoms were severe enough to warrant a reoperation.

\section{TABLE 3. Radiographic patterns of ASD*}

\begin{tabular}{lcc}
\hline \multicolumn{1}{c}{ Patterns of ASD } & Group A $(n=20)$ & Group B $(n=3)$ \\
\hline Retrolisthesis & 9 & 0 \\
\hline Retrolisthesis + DD & 6 & 1 \\
\hline $\mathrm{DD}$ & 1 & 2 \\
\hline Anterolisthesis & 3 & 0 \\
\hline Anterolisthesis + DD & 1 & 0 \\
\hline DD = disc degeneration with or without intradiscal vacuum clefts or endplate \\
changes. \\
* Diagnoses were based on lateral flexion/extension radiographs. Retrolis- \\
thesis and anterolisthesis were associated increased segmental mobility as \\
compared to preoperative status.
\end{tabular}

TABLE 4. Preoperative Pfirrmann status of the segment immediately proximal to PLIF

\begin{tabular}{ccccccc}
\hline & \multicolumn{2}{c}{ Group A $(n=42)$} & & \multicolumn{2}{c}{ Group B $(n=49)$} \\
\cline { 2 - 3 } \cline { 6 - 7 } Pfirrmann Grade & Preop & ASD & & Preop & ASD \\
\hline I & 9 & 1 & & 6 & 0 \\
\hline II & 25 & 16 & & 16 & 0 \\
\hline III & 6 & 3 & & 16 & 1 \\
\hline IV & 2 & 0 & & 11 & 2 \\
\hline V & 0 & 0 & & 0 & 0 \\
\hline
\end{tabular}

\section{Clinical Outcome}

In both groups, significant pain relief and functional improvement were observed following surgery. The mean back and leg VAS scores and ODI data are summarized in Table 6. At 24 months, the outcomes were worse in Group A than in Group B, as reflected by significantly higher VAS and ODI. However, at the final follow-up evaluations, after reoperation to treat some symptomatic ASD cases, no significant differences in these parameters existed between the 2 groups (Table 6).

\section{Complications and Comorbidities}

There was no surgery-related mortality. No spinous process fracture occurred in Group B during DIAM implantation. A small dural tear occurred in 1 Group B patient while the DIAM implant was placed. It was closed primarily. One Group B patient had deep vein thrombosis and another had acute myocardial infarction during their postoperative hospital stay. Both received medical interventions, and the latter patient also underwent coronary stent placement. One patient in each group had a superficial wound infection that required additional wound care and antibiotic therapy. Comorbidities diagnosed during follow-up included Alzheimer's disease in 1 patient and thyroid cancer in 1 patient in Group A and Parkinson's disease in 4 patients and breast cancer in 2 patients in Group B. All of these patients received appropriate medical and surgical care for their conditions. These diseases did not affect the evaluation of clinical outcomes in these patients.

\section{Discussion}

In this study, we excluded cases in which PLIF was

TABLE 5. Preoperative Pfirrmann status of DIAM-implanted segments in Group B

\begin{tabular}{ccc}
\hline \multirow{2}{*}{$\begin{array}{c}\text { Pfirrmann Grade } \\
(n=49)\end{array}$} & \multicolumn{2}{c}{ Final Flexion/Extension Mobility } \\
\cline { 2 - 3 }$I(n=6)$ & 5 & 1 \\
\hline$I I(n=16)$ & 15 & 1 \\
\hline$I I I(n=16)$ & 9 & $7(1$ ASD $)$ \\
\hline$I V(n=11)$ & 6 & $5(2$ ASDs $)$ \\
\hline$V(n=0)$ & 0 & 0 \\
\hline${ }^{*}$ Of the 14 patients showing restricted or no mobility at final follow-up, 5 had \\
already lost mobility at the DIAM-implanted segment before surgery.
\end{tabular}


TABLE 6. Clinical outcomes

\begin{tabular}{cccc}
\hline Variable & Group A $(n=42)$ & Group B $(n=49)$ & p Value \\
\hline Back pain VAS & & & \\
\hline Preop & $7.0 \pm 1.2$ & $7.1 \pm 1.4$ & 0.551 \\
\hline 24 mos & $2.5 \pm 2.8$ & $1.3 \pm 2.3$ & 0.036 \\
\hline Final $^{*}$ & $1.5 \pm 2.1$ & $1.5 \pm 2.4$ & 0.678 \\
\hline Leg pain VAS & & \\
\hline Preop & $7.1 \pm 1.4$ & $7.2 \pm 1.3$ & 0.571 \\
\hline 24 mos & $2.6 \pm 2.4$ & $1.4 \pm 2.5$ & 0.035 \\
\hline Final & $1.5 \pm 2.2$ & $1.4 \pm 2.3$ & 0.702 \\
\hline ODI score & & & \\
\hline Preop & $26.3 \pm 3.4$ & $27.7 \pm 3.8$ & 0.632 \\
\hline 24 mos & $18.9 \pm 4.0$ & $14.6 \pm 3.4$ & 0.037 \\
\hline Final & $15.5 \pm 4.2$ & $14.1 \pm 3.9$ & 0.220 \\
\hline
\end{tabular}

* Final data represent assessments made at the last follow-up visits. For patients who received a second operation for symptomatic ASD, data indicate results recorded after reoperations.

performed without involving the L5-S1 segment for 2 reasons. First, as far as clinical evaluation of ASD is concerned, including the L5-S1 level would eliminate any distal ASD, which may cause confounding symptoms. Second, the anatomical features of the sacrum (small and short spinous processes) preclude secure anchoring of the DIAM implant at the L5-S1 interspinous space. Therefore, we chose to address only ASD proximal to PLIF.

\section{Clinical Relevance}

Following lumbar fusion, hypermobility ${ }^{1}$ and disc height reduction ${ }^{21}$ can be observed at the proximal adjacent segments. Although not necessarily associated with symptoms, these changes may reflect increased segmental biomechanical stress and may eventually lead to ASD. ${ }^{17}$ The risk factors predisposing to symptomatic ASD include age older than 60 years, preexisting degeneration, multilevel fusions, and fusions adjacent to but not including the L5-S1 level. ${ }^{13}$ Undeniably, many patients who need lumbar fusion are elderly, require multilevel fusion for advanced degenerative disease, and are at risk of developing ASD for these reasons. Therefore, any measures that are effective in preventing ASD, and thus reducing the possibility of future reoperation, would be meaningful and beneficial, even if it is not known who will become symptomatic. Such a notion is supported by the current study, which shows a relatively high rate of radiographic ASD becoming symptomatic once it developed ( 9 of 20 cases in Group A).

In our series, the most common pattern of ASD was retrolisthesis with or without disc degeneration, which accounted for three-fourths of the cases of radiographic ASD in Group A. Given the finding that PLIF surgery significantly reduced lumbar lordotic angles in both groups, it can be speculated that retrolisthesis may well be a compensatory biomechanical component that develops as a result of the relative kyphosis created by PLIF. This finding is in line with a previous study showing retrolisthesis as the most frequently observed pattern of ASD. ${ }^{11}$ One of the biomechanical features of IPDs is to act as a spinal extension block..$^{18}$ This appears to be an advantage for preventing retrolisthesis, which is usually most prominent on extension (Fig. 2D). Therefore, one major mechanism of DIAM-related prevention of ASD may be through the inhibition of hypermobility associated with retrolisthesis.

There may be concerns that an IPD may lead to the development of ASD due to the restriction of segmental mobility. Our results show that although the DIAM implant prevented hypermobility, it did not make the implanted segment fixed. In 35 (71\%) of the 49 patients in Group $\mathrm{B}$, segmental flexion/extension mobility of the DIAM segments was preserved. This may explain why there was no ASD proximal to the DIAM levels in our series. Even in patients showing limited or no flexion/extension mobility at the DIAM-implanted segments, the proximal adjacent segments remained stable. These findings are in accordance with a recent study showing that the DIAM implant does not significantly affect the range of motion of the proximal and distal adjacent segments. ${ }^{5}$
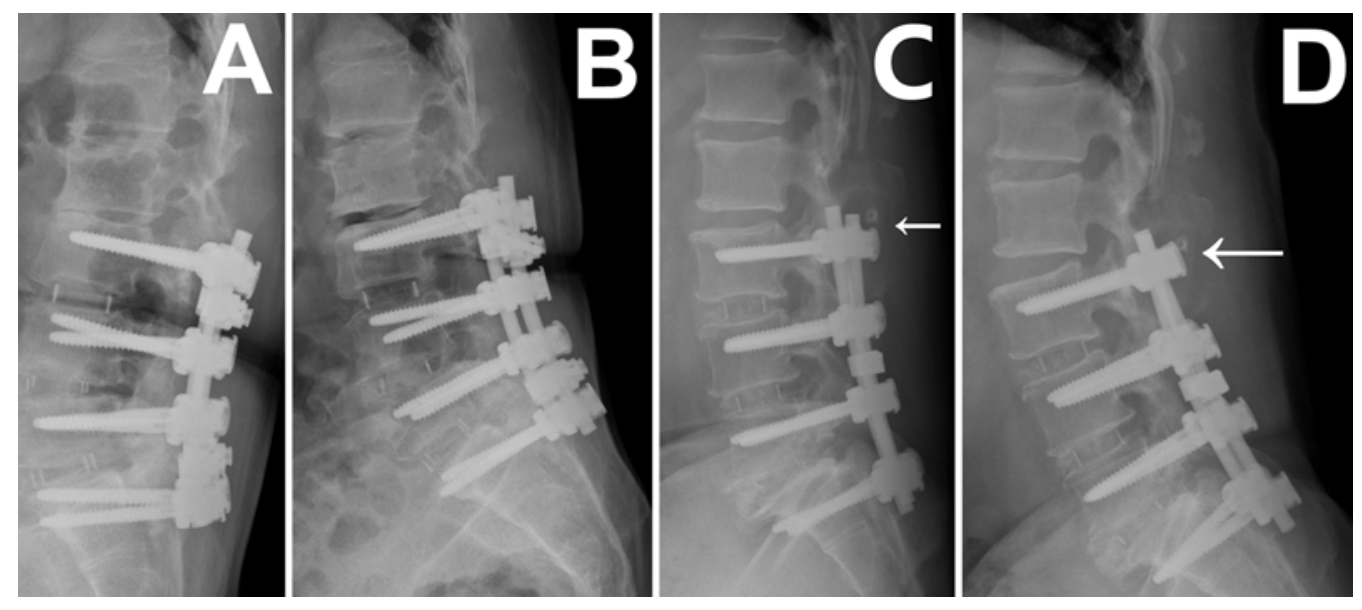

FIG. 2. A and B: Lateral flexion/extension radiographs obtained 34 months after PLIF showing ASD at the proximal 2 levels in a 57-year-old Group A patient. C and D: Images obtained in a 59-year-old Group B patient at 36 months after PLIF showing maintenance of disc height with segmental flexion/extension mobility at the proximal adjacent segment in the presence of a DIAM implant (arrow), despite a tendency for retrolisthesis. 
Our data suggest that if the preoperative disc status of the DIAM-implanted level is Pfirrmann Grade I or II, segmental flexion/extension mobility tends to be preserved. In contrast, if it is Grade III or IV, mobility becomes restricted or even lost. It is also to be noted that of the 3 patients with ASD in Group B, 1 had Grade III disc status and 2 had Grade IV disc status prior to DIAM implantation (Tables 4 and 5). These findings seem to imply that the initial disc status may be an important factor predicting the future outcome after DIAM implantation, and hence the need to scrutinize the applicability when the segment to be implanted has already been rather degenerated.

Clinical outcome measurements recorded at 24 months after surgery showed that Group A fared worse than Group $\mathrm{B}$ at this time point. The finding was due to the presence of significantly more severe symptoms in the patients with symptomatic ASD in Group A, and it provides evidence supporting the potential benefit of DIAM implantation in Group B. At the final follow-up evaluations, after reoperations had been performed and the patients had recovered, outcome measurements became similar in the 2 groups (Table 6).

\section{Limitations and Bias}

For stable DIAM anchoring, the spinous processes and laminae of the most cephalad PLIF segment must be at least partially preserved and the attached supraspinous ligament kept intact. Maintaining the integrity of these structures in fusion surgery has been proposed as a measure to prevent ASD. ${ }^{3,12}$ It can therefore be argued that the low incidence of ASD in Group B was related to the structural preservation itself. To minimize the bias of unequal bone resection between the 2 groups, we only included Group A cases in which similar limited bone resection at the uppermost PLIF segment was performed. Even so, however, minor differences in bone resection might still have existed between the groups, since the extent of resection might have varied due to stenosis severity and intraoperative judgment.

The condition of the disc proximal to the treated levels was Pfirrmann Grade I or II in 34 (81\%) of the 42 Group A patients, whereas in 27 (55\%) of the 49 Group B patients it was Pfirrmann Grade III or IV (Table 4). This difference implies that once the decision for DIAM implantation was made, the surgeon tended to put the implant at a segment that would otherwise be considered for fusion. While it suggests bias in decision making, it again consolidates the evidence for a beneficial effect of proximal DIAM implantation, as Group B patients had more degenerative adjacent discs proximal to fusion to begin with, but ended up with a much lower rate of ASD, compared with Group A.

The mean age of Group B patients $(64.5 \pm 7.2$ years) was found to be greater than that of Group A patients (59.1 \pm 8.6 years $)(\mathrm{p}=0.001)$. This might have been due to the presence of selection bias, as the surgeon might have been more aggressive in proposing DIAM implantation to older patients, anticipating higher risk for future ASD. However, this difference in the ages of the patients should not be regarded as a shortcoming. On the contrary, it should be viewed as evidence supporting the effectiveness of DIAM implantation, since Group B patients had a significantly lower incidence of ASD despite their older age, which is a risk factor for ASD. ${ }^{13}$

Finally, the present study did not address the mechanism, benefits, and cost effectiveness of adding the DIAM implant proximal to multilevel PLIF as a strategy for preventing ASD. There is no doubt that these are important issues warranting further investigation.

\section{Conclusions}

Creating a dynamic transition zone rostral to a multilevel PLIF construct with the DIAM implant was associated with a significant decrease in the occurrence of radiographic ASD as well as lower rates of symptomatic ASD and reoperation. In the majority of DIAM-implanted segments, disc status and normal flexion/extension mobility were maintained. Furthermore, DIAM implantation itself did not cause ASD proximal to the implant. However, clinical success may depend on careful evaluation so that a sound decision can be made as to whether a segment should be fused or suitable to act as the dynamic transition zone. Further controlled studies are needed to clarify the long-term benefit and outcome, and cost effectiveness of such a strategy.

\section{References}

1. Bastian L, Lange U, Knop C, Tusch G, Blauth M: Evaluation of the mobility of adjacent segments after posterior thoracolumbar fixation: a biomechanical study. Eur Spine J 10:295-300, 2001

2. Cheh G, Bridwell KH, Lenke LG, Buchowski JM, Daubs MD, Kim Y, et al: Adjacent segment disease following lumbar/thoracolumbar fusion with pedicle screw instrumentation: a minimum 5-year follow-up. Spine (Phila Pa 1976) 32:2253-2257, 2007

3. Chen LH, Lai PL, Tai CL, Niu CC, Fu TS, Chen WJ: The effect of interspinous ligament integrity on adjacent segment instability after lumbar instrumentation and laminectomyan experimental study in porcine model. Biomed Mater Eng 16:261-267, 2006

4. Etebar S, Cahill DW: Risk factors for adjacent-segment failure following lumbar fixation with rigid instrumentation for degenerative instability. J Neurosurg 90 (2 Suppl):163-169, 1999

5. Ha KY, Seo JY, Kwon SE, Son IN, Kim KW, Kim YH: Posterior dynamic stabilization in the treatment of degenerative lumbar stenosis: validity of its rationale. J Neurosurg Spine 18:24-31, 2013

6. Hasz MW: Diagnostic testing for degenerative disc disease. Adv Orthop 2012:413913, 2012

7. Imagama S, Kawakami N, Matsubara Y, Kanemura T, Tsuji T, Ohara T: Preventive effect of artificial ligamentous stabilization on the upper adjacent segment impairment following posterior lumbar interbody fusion. Spine (Phila Pa 1976) 34:2775-2781, 2009

8. Kanayama M, Hashimoto T, Shigenobu K, Harada M, Oha F, Ohkoshi Y, et al: Adjacent-segment morbidity after Graf ligamentoplasty compared with posterolateral lumbar fusion. J Neurosurg 95 (1 Suppl):5-10, 2001

9. Korovessis P, Repantis T, Zacharatos S, Zafiropoulos A: Does Wallis implant reduce adjacent segment degeneration above lumbosacral instrumented fusion? Eur Spine J 18:830-840, 2009

10. Kumar A, Beastall J, Hughes J, Karadimas EJ, Nicol M, Smith F, et al: Disc changes in the bridged and adjacent seg- 
ments after Dynesys dynamic stabilization system after two years. Spine (Phila Pa 1976) 33:2909-2914, 2008

11. Kumar MN, Baklanov A, Chopin D: Correlation between sagittal plane changes and adjacent segment degeneration following lumbar spine fusion. Eur Spine J 10:314-319, 2001

12. Lai PL, Chen LH, Niu CC, Fu TS, Chen WJ: Relation between laminectomy and development of adjacent segment instability after lumbar fusion with pedicle fixation. Spine (Phila Pa 1976) 29:2527-2532, 2004

13. Lawrence BD, Wang J, Arnold PM, Hermsmeyer J, Norvell DC, Brodke DS: Predicting the risk of adjacent segment pathology after lumbar fusion: a systematic review. Spine (Phila Pa 1976) 37 (22 Suppl):S123-S132, 2012

14. Lee CH, Hyun SJ, Kim KJ, Jahng TA, Yoon SH, Kim HJ: The efficacy of lumbar hybrid stabilization using the DIAM to delay adjacent segment degeneration: an intervention comparison study with a minimum 2-year follow-up. Neurosurgery 73 (2 Suppl Operative):ons224-ons232, 2013

15. Lee CK: Accelerated degeneration of the segment adjacent to a lumbar fusion. Spine (Phila Pa 1976) 13:375-377, 1988

16. Lund T, Oxland TR: Adjacent level disk disease-is it really a fusion disease? Orthop Clin North Am 42:529-541, viii, 2011

17. Park P, Garton HJ, Gala VC, Hoff JT, McGillicuddy JE: Adjacent segment disease after lumbar or lumbosacral fusion: review of the literature. Spine (Phila Pa 1976) 29:19381944, 2004

18. Park SW, Lim TJ, Park J: A biomechanical study of the instrumented and adjacent lumbar levels after In-Space in- terspinous spacer insertion. J Neurosurg Spine 12:560-569, 2010

19. Pfirrmann CW, Metzdorf A, Zanetti M, Hodler J, Boos N: Magnetic resonance classification of lumbar intervertebral disc degeneration. Spine (Phila Pa 1976) 26:1873-1878, 2001

20. Resnick DK, Choudhri TF, Dailey AT, Groff MW, Khoo L, Matz PG, et al: Guidelines for the performance of fusion procedures for degenerative disease of the lumbar spine. Part 4: radiographic assessment of fusion. $\mathbf{J}$ Neurosurg Spine 2:653-657, 2005

21. Schulte TL, Leistra F, Bullmann V, Osada N, Vieth V, Marquardt $\mathrm{B}$, et al: Disc height reduction in adjacent segments and clinical outcome 10 years after lumbar 360 degrees fusion. Eur Spine J 16:2152-2158, 2007

\section{Author Contributions}

Conception and design: Lu. Acquisition of data: Lu, Liliang, HK Wang, JS Chen, KW Wang. Analysis and interpretation of data: $\mathrm{Lu}$, Liliang, Liang. Drafting the article: Lu. Approved the final version of the manuscript on behalf of all authors: Lu. Statistical analysis: Liang, TB Chen. Study supervision: Lu, HJ Chen. Performed surgical procedures: Lu.

\section{Correspondence}

Kang Lu, Department of Neurosurgery, E-Da Hospital, College of Medicine, I-Shou University, 1 Yida Rd., Yanchau District, Kaohsiung 824, Taiwan. email: kanglu.1002@gmail.com. 